العوامل المحدة للطلب علي اللحوم البيضاء في مصر

\author{
داليا حامد الثويخ'، حسن موسى رضوان' \\ 'قسم الاقتصاد الزراعي - كلية الزراعة - جامعة أسيوط

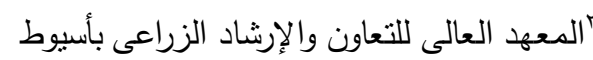

الملخص العربي

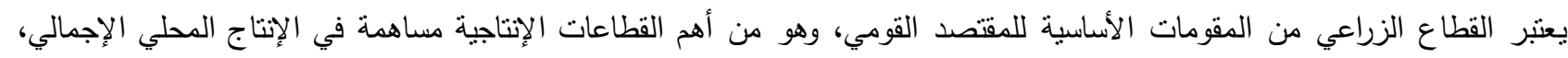

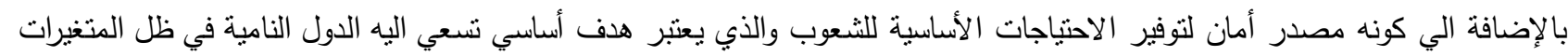

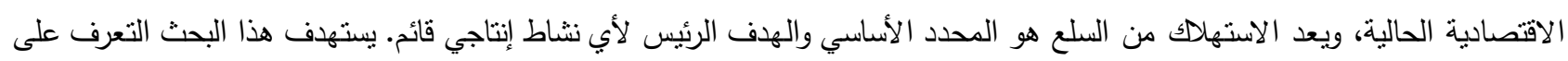

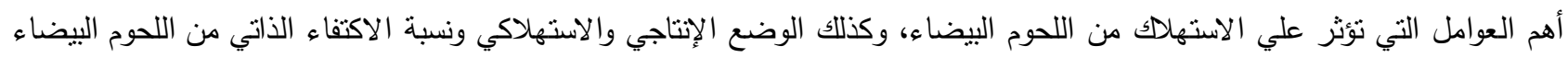

في مصر . توصل البحث لعدة نتائج كما يلي:

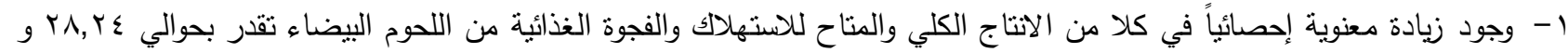

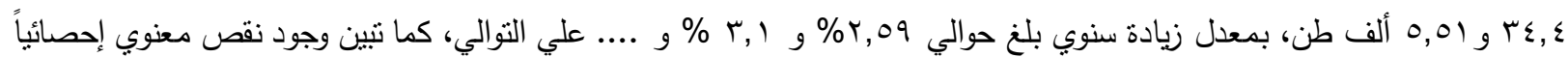

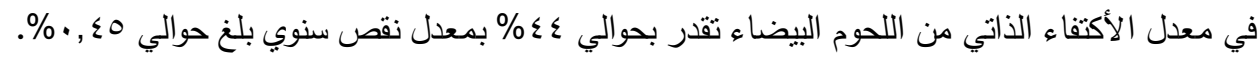

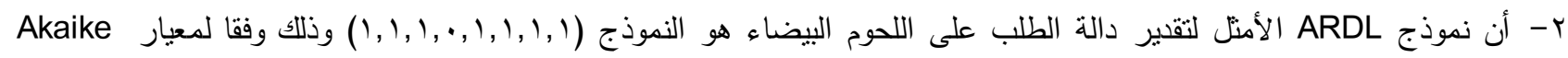
information criteria

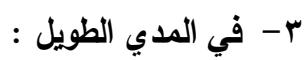
• بلغت مرونة الطلب السعرية للاواجن حوالى -10.. وهى سالبة وأقل من الواحد الصحيح مما يدل على أن الدواجن من السلع الضرورية بالنسبه للمستهلاك لتلبية الأحتباجات من البروتين الحيوانى. بلغت مرونة الطلب التقاطعية بالنسبة لسعر التجزئه الحقيقى للاسماك حوالى حب . . ، ،أى ان زياده سعر التجزئه الحقيقى للاسماك بمقدار

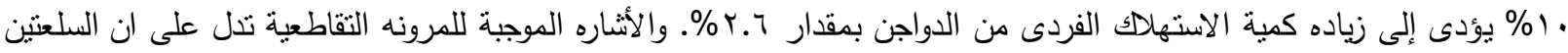

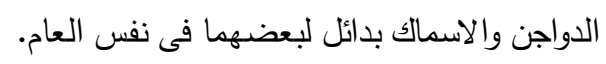

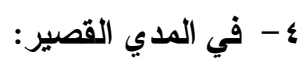

بلغت مرونة الطلب التقاطعية بالنسبة لسعر التجزئه الحقيقي للحوم الحمراء العام الحالي حوالى عا.. ، أع ان زياده سعر التجزئه

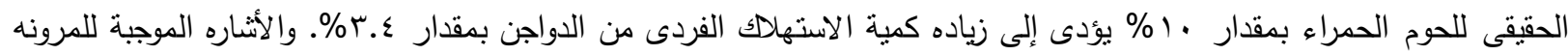

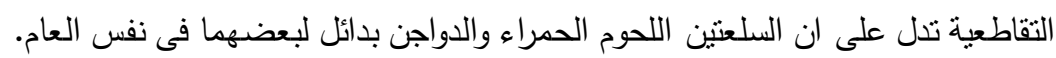

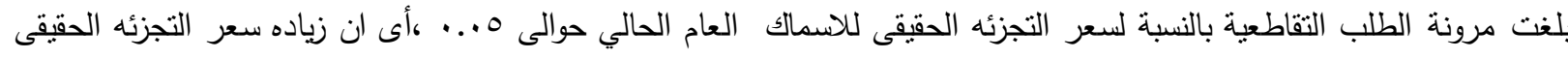

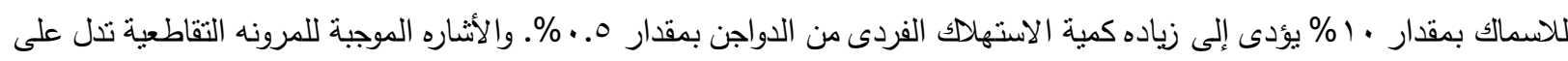

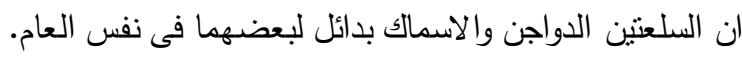

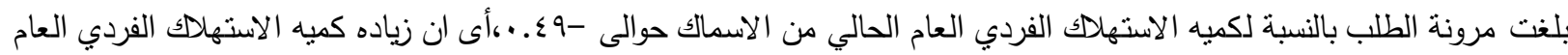

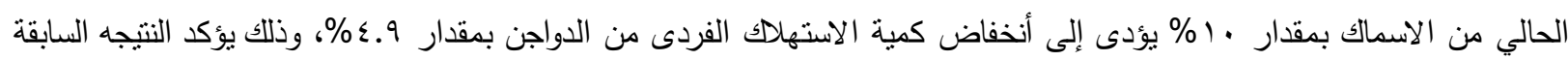

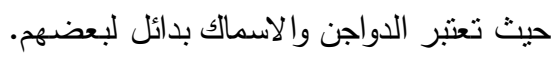
بلغت مرونة الطلب بالنسبة لسعر أستيراد الدواجن العام الحالى حوالى -1 ... . ، مما يدل على أنه طلب غير مرن حيث أن زيادة سعر

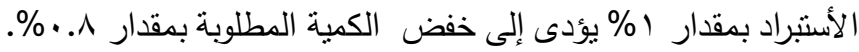


الاستعانة بأستخدام أساليب الاقتصاد القياسي لتقلير دالة الطلب على الإلى

اللحوم البيضاء في الأجل القصبر والأجل الطويل كنموذج الانحدار الذاني ذو الفجوات الزمنية الموزعة والمعروف بـ (ARDL) وهو الاجل نموذج يفسر العلاقة الديناميكية بين المتغير التابع والقيم السابقة

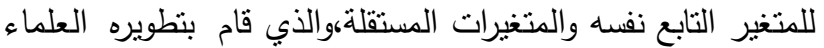
Pesaran, Shin, Smith الحدود Bounds test كأحد خطوات نموذج (ARDL) ويختبر علاقات التكامل المشترك بدون شرط قيد التكامل من نفس الرتبة اللسلاسل موضع الدراسة، وذلك بعد أختبار أستقرار السلاسل الزمنية موضع الدراسة من خلال اختبارات جذر الوحدة كاختبار ديكي فوللر

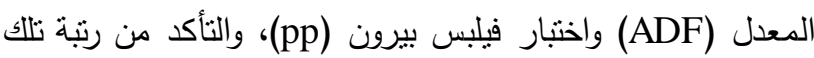
السلاسل الزمنية التى تثفق مع شروط النموذج المنبع فى عملية

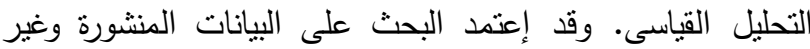
المنشورة التي تصدرها وزارة الزراعة واستصلاح الأراضي و الجهاز

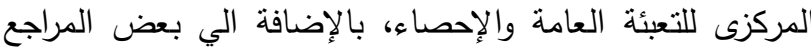
والدراسات السابقة والتى تخدم هذا البحث.

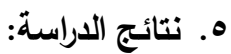

هـ الأهمية النسبية لقيمة الانتاج الاجني من إجمالي قيمة الإنتاج الززاعي والحيواني في مصر.

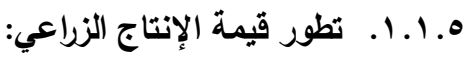

نتير البيانات الواردة في الجدول رقم (1) إلى تطور قيمة

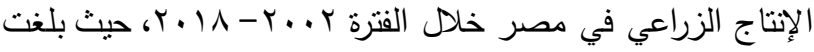

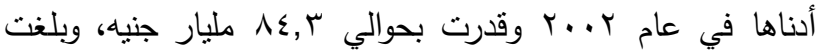

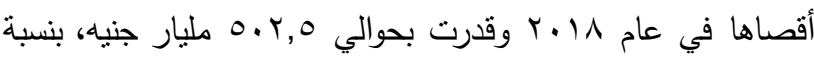

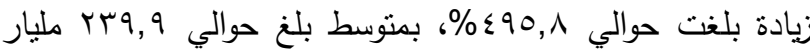
جنيه خلال الفتزة موضع الدراسة. وبتقلير معادلة الإتجاه الزمني

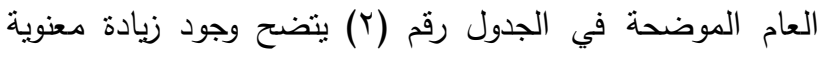

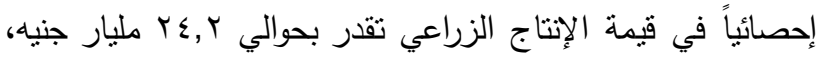

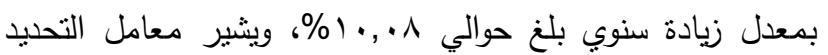
(ر r) إلي أن حوالي ب9\% من التغيرات الحاثثة تعزي إلي العوامل

$$
\text { التي يعكسها عنصر الزمن. }
$$

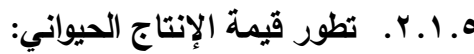

نتير الييانات الواردة في الجدول رقم (1) إلى تطور قيمة

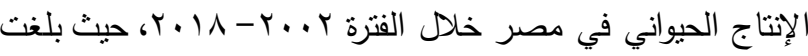

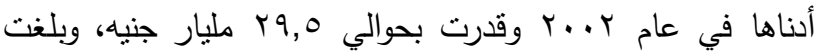

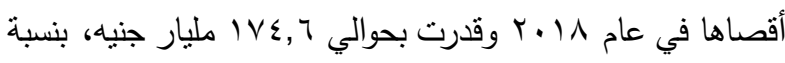

يعثبر القطاع الزراعي من المقومات الأساسية للمقتصد

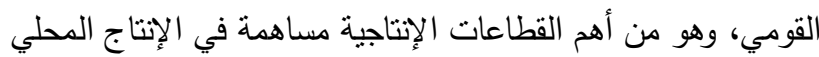
الإجمالي، بالإضافة الي كونه مصدر أمان لتوفير الاحتياجات الأساسية للثعوب والذي يعتبر هدف أساسي تسعي اليه الدول النامية

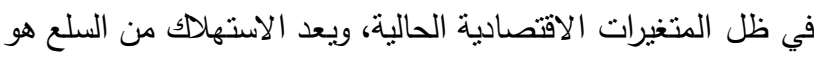
المحدد الأساسي والهدف الرئيس لأي نشاط إنتاجي قائم (ّأ. تتميز الدواجن بارتفاع قيمتها الغذائية ورخص ثمنها مقارنة باللحوم الحمراء ، وارتفاع معامل التحويل الغذائي لها ـ كما تتميز

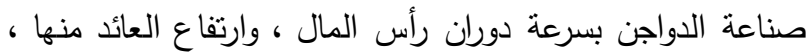

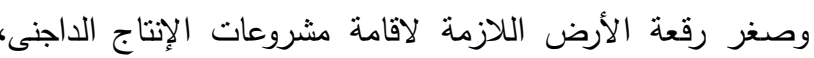

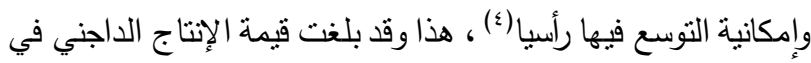

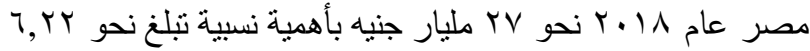

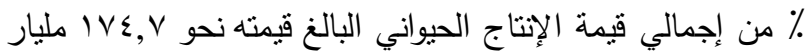

$$
\text { جنيه (') ( ) }
$$

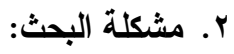

تكمن مشكلة البحث في إنخفاض منوسط نصيب الفرد من

البروتين الحيواني في مصر، وعلي الرغم من الجهود التي تنذلها

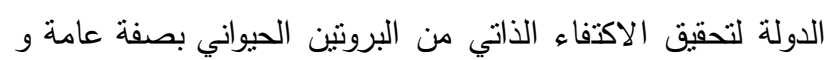

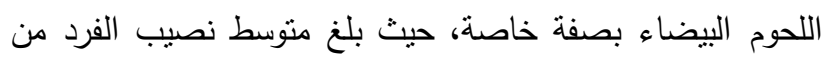

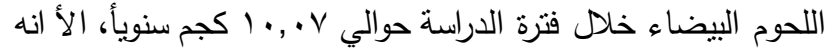

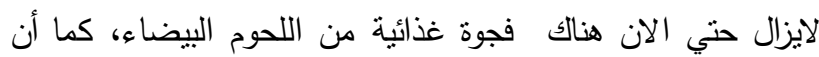

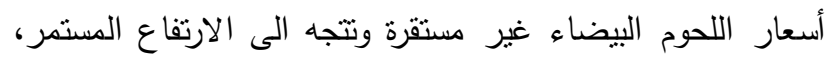

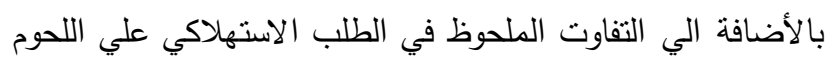
اليضناء بين الطبقات الاجتماعية المختلفة في مصر.

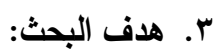

يستهدف هذا البحث دراسة الوضع الإنتاجي والاستهلاكي ونسبة الاكتفاء الذاتي من اللحوم الليضاء في مصر خلاد الفترة

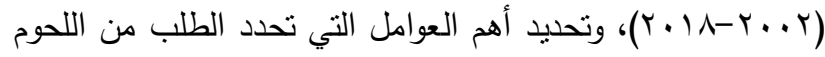
الييضاء فى مصر في الأجل القصبروالطويل خلا الفترة من (r.)1 $(1990)$ ؛. الأسلوب البحثي و مصادر البيانات:

إعتمد البحث على استخدم إسلوب التحليل الإحصائي الوصفي والاستدلالي لتحقيق أهداف البحث، من خلال استخدام بعض الأساليب الرياضية والاحصائية مثل المتوسطات الحسابية والنسب المئوية وتقبير معادلات الاتجاه الزمنى العام، كما تم 


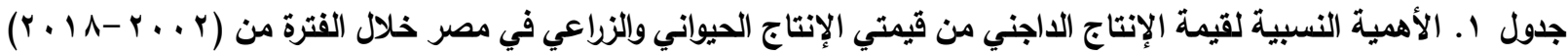
القيمة: ( مليون جنيه)

\begin{tabular}{|c|c|c|c|c|c|}
\hline \% قيمة الإنتاج & \% قيمة الإنتاج & قالدة الإنتاج & قيمة الإنتاج & قالزمة الإنتاج & السنوات \\
\hline YI.TV & $V . \varepsilon r$ & 7YT7 & rq $0 V$ & $\Lambda \leqslant Y V$. & $r \ldots r$ \\
\hline 11.00 & 7.71 & $T \varepsilon \cdot T$ & $r \leqslant 0.7$ & 9710r & $r \ldots r$ \\
\hline 19.40 & T.VA & VONV & rq199 & סזגו & $r \ldots \varepsilon$ \\
\hline س & 7.1. & $V V \leqslant V$ & $\Sigma \vee \backslash r$. & |r79v| & $r \ldots o$ \\
\hline $1 \leq . \leq \Lambda$ & O.Y & VIAY & $\leq 90 \vee 1$ & $1 r V \leqslant 19$ & $r \ldots T$ \\
\hline $10 . Y \varepsilon$ & $0 . \Lambda$ & $\Lambda \varepsilon \cdot \varepsilon$ & $001 r q$ & $1009 \leqslant 0$ & $r \ldots v$ \\
\hline $10.9 \mathrm{~V}$ & 0.01 & 1.r. & $T \leq 9 \leq$. & $11074 \mathrm{~V}$ & $r \ldots \lambda$ \\
\hline $17 . .9$ & 0.17 & 111.7 & 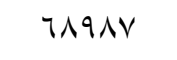 & $1 \wedge 9 \leqslant 41$ & $r \ldots q$ \\
\hline 17.91 & $T . Y \varepsilon$ & V T T & VVYor & r.qTOS & $r \cdot 1$. \\
\hline $1 V .00$ & $0.9 \pi$ & $1 \leqslant \Lambda \leqslant r$ & 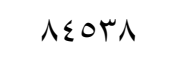 & $r \leq 99 \wedge 9$ & $r .11$ \\
\hline 11.01 & $7.1 \mathrm{~V}$ & 170.1 & גMNץT & rTV $\{Y \leq$ & r.IT \\
\hline TY.MT & v.vi & rivar & $q V Y \leq r$ & rAY $\leqslant T_{0}$ & $r .1 r$ \\
\hline TY.IT & ᄉ.11 & Y $\leqslant \vee \wedge T$ & $11 r \cdot r q$ & $r .0 \leqslant 1 \leqslant$ & $r \cdot 1 \varepsilon$ \\
\hline TY.TO & ᄉ. $\leqslant 0$ & $r V \cdot r T$ & $119 \mathrm{rVq}$ & $r 190 \leqslant 9$ & $r .10$ \\
\hline MY.OS & A.YV & $\Gamma \cdot 1 \wedge \varepsilon$ & & $r ฯ \leqslant \wedge \leqslant 0$ & $r .17$ \\
\hline rT. & V.VT & $r \neg \leqslant V \leqslant$ & 171099 & EVIVIT & r.IV \\
\hline ז.... & 1.99 & $\leq 7 \lambda) \leqslant$ & $I V \leqslant T V V$ & Or. $\leqslant$ Vo & $r \cdot 1 \Lambda$ \\
\hline 19,r & $7, \wedge 0$ & $\mid V \varepsilon \varepsilon \varepsilon$ & $10 . \leqslant$. & r T৭q४A & المتوسط \\
\hline
\end{tabular}
جدول r. نتائج تقدير الاتجاه الزمني العام للأهمية النسبية لقيمة الإنتاج الاجني من قيمتي الإنتاج الحيواني والزراعي في مصر خلال

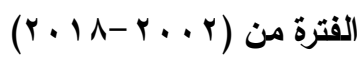

\begin{tabular}{|c|c|c|c|c|}
\hline معدل التغير السنوي(\%) & ف & rر & المعادلة & البيان \\
\hline $1 \cdot, \cdot 1$ & $"$ I $v 0,0$ & $\cdot, 9 r$ & 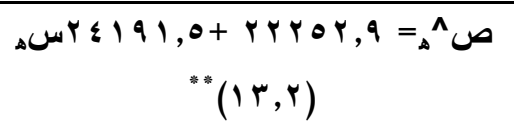 & قيمة الانتاج الزراعي \\
\hline 1. & $" * * 1 \wedge, q$ & $\cdot 94$ & 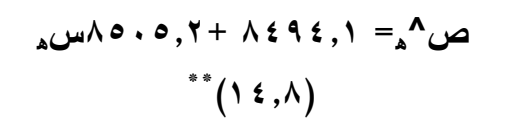 & قيمة الانتاج الحيواني \\
\hline Ir,o & ${ }^{* *} \wedge V, \cdot r$ & $\cdot, \wedge \bullet$ & 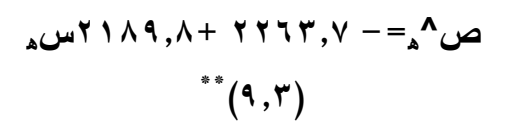 & قيمة الانتاج الداجني \\
\hline \multicolumn{5}{|c|}{ ** معنوي عند مستوي المعنوي ا +.. } \\
\hline
\end{tabular}


الموضحة في الجدول رقم (ع) يتضح وجود زيادة معنوية إحصائياً

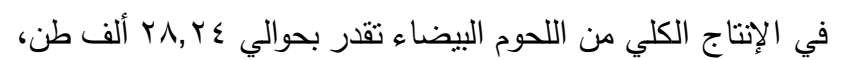

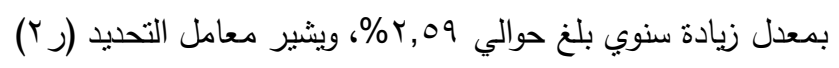

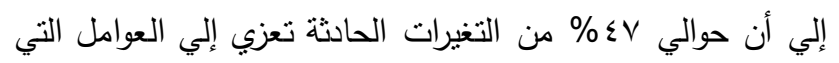

$$
\text { يعكسها عنصر الزمن. }
$$

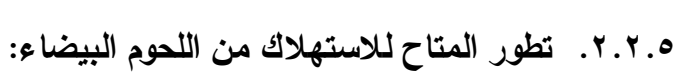

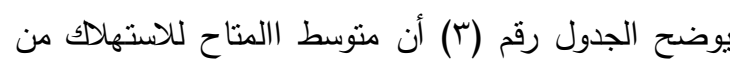

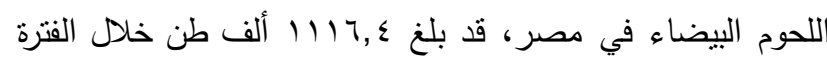

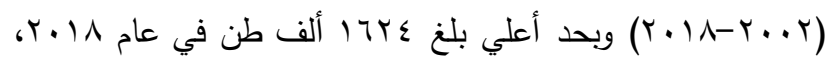

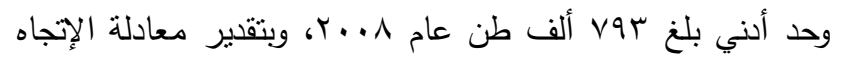

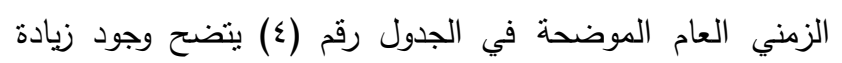

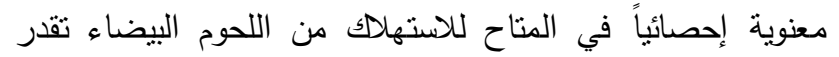

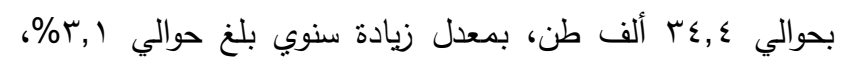

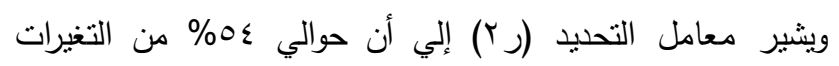
الحادثة تعزي إلي العوامل التي يعكسها عنصر الزمن.

ـ.Y.Y. ت تطور الفجوة الغذائية من اللحوم البيضاء في مصر: يوضح الجدول رقم (ץ) أن متوسط الفجوة الغذائية من اللحوم

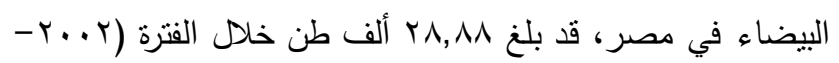

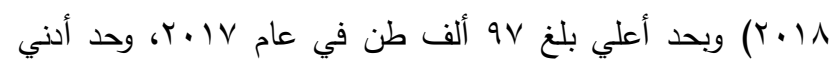

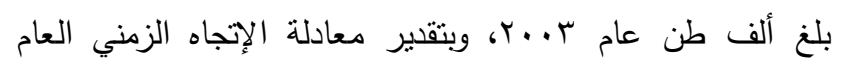
الموضحة في الجدول رقم (ع) يتضح وجود زيادة معنوية إحصائياً

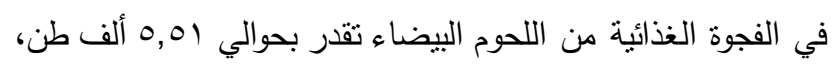

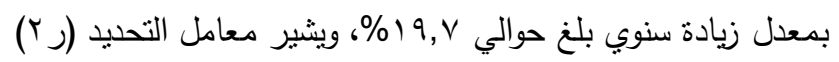

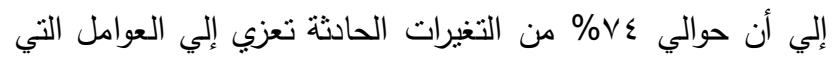

$$
\text { يعكسها عنصر الزمن. }
$$

ه.r.؟. ت تطور معدل الأكتفاء الأتي من اللحوم البيضاء:

يوضح الجدول رقم (ץ) أن منوسط معدل الأكتفاء الذاتي من

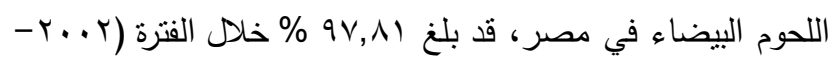

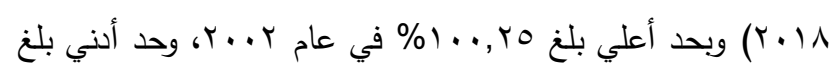

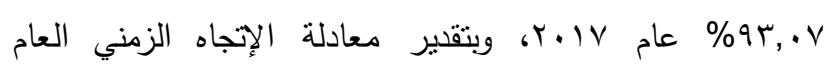
الموضحة في الجدول رقم (ع) يتضح وجود نقص معنوية إحصائياً

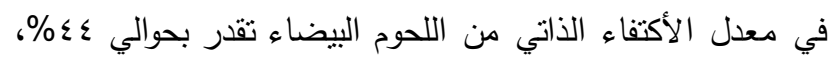

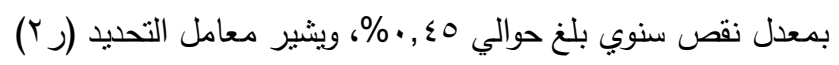

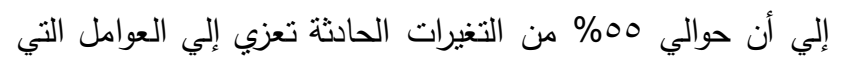
يعكسها عنصر الزمن.

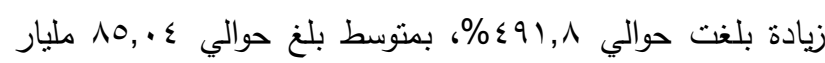

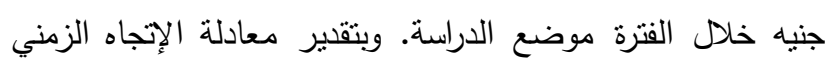
العام الموضحة في الجدول رقم (r) يتضح وجود زيادة معنوية

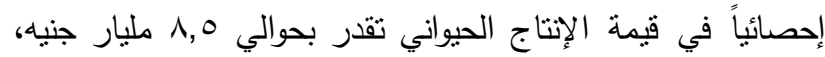

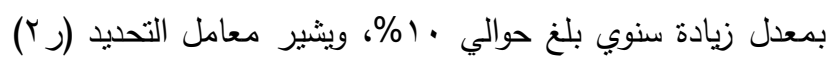

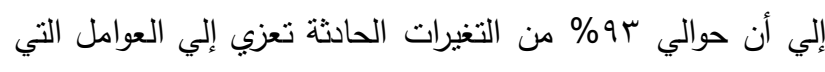

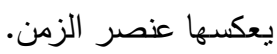

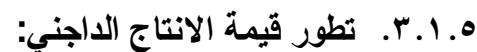

تشير الليانات الواردة في الجدول رقم (1) إلى نطور قيمة

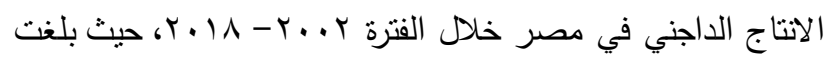

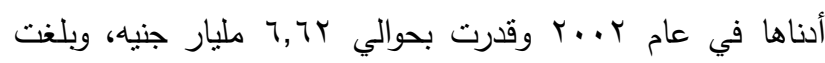

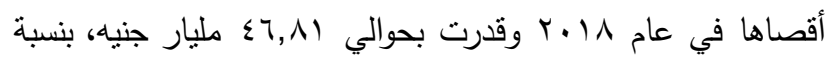

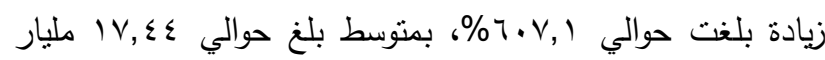

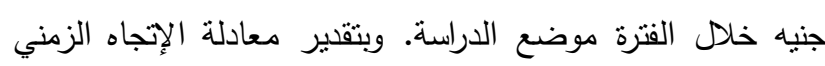
العام الموضحة في الجدول رقم (Y) بيضح وجود زيادة معنوية

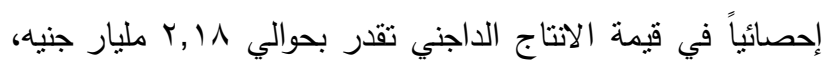

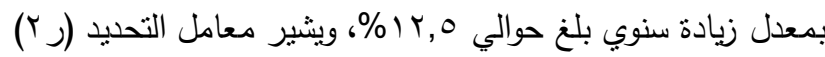

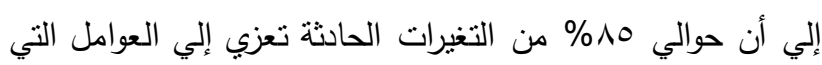
بعكسها عنصر الزمن.

هـ ا.ـ. تطور الأهمية النسبية لقيمة الانتاج الداجني: تثير الييانات الواردة في الجدول رقم (1) إلى تطور الأهمية

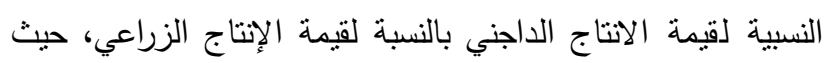

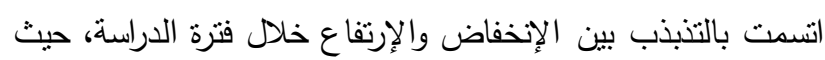

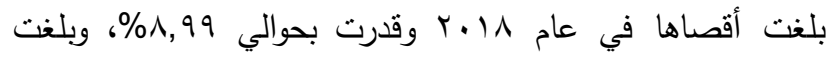

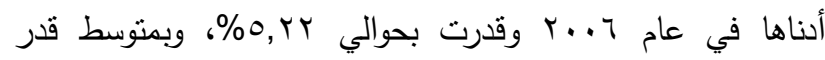

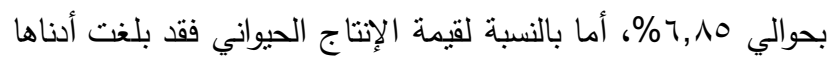

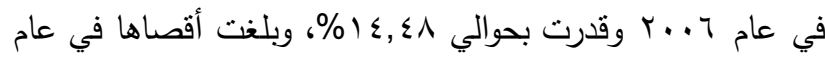

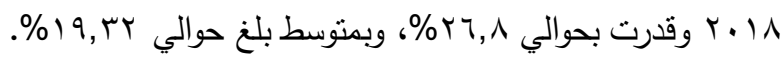
ه.Y. مؤثرات إنتاج واستهلاك اللحوم البيضاء ونسبة الاكتفاء الاتي في مصر: مؤثرات إنتا: ه.r. 1. . تطور الإنتاج الكلي من اللحوم البيضاء في مصر : يوضح الجدول رقم (r) أن منوسط الاتتاج الكلي من اللحوم

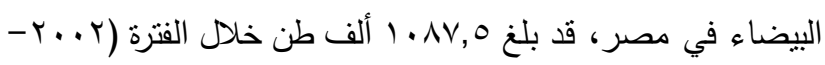

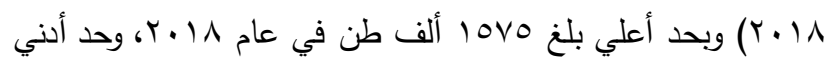

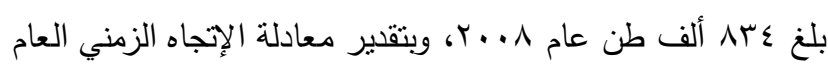


Dalia Hamed El Showeikh and Hassan Mousa. Radwan., 2021

جدول r. تطور كمية الانتاج الكلي والمتاح للاستهلاك والفجوة الغذائية ونسبة الاكتفاء الذاتي من اللحوم البيضاء في مصر خلال الفترة

\begin{tabular}{|c|c|c|c|c|c|}
\hline & & & & & $\cdot r)$ \\
\hline متوسط نصيب الفرد & \% الاكتفاء الذاتي & (الفجوة الغذائية & (المتاح للاستهلاك & (الفتاج الكلي & السنوات \\
\hline Ir.r & $1 . . r_{0}$ & $r$ & 1171 & $117 \varepsilon$ & $r \ldots r$ \\
\hline $11 . r$ & $1 \ldots 1$ & 1 & $1 . \leqslant V$ & $1 . \leqslant 1$ & $r . . r$ \\
\hline 11.1 & 99.19 & $1-$ & q৯r & $9 \wedge r$ & r... \\
\hline $1 . . v$ & $1 \ldots .19$ & r & 1.17 & 1.11 & r...o \\
\hline V.A & $91 . \vee 0$ & $1 .-$ & 1.0 & 190 & $r \ldots T$ \\
\hline$\Lambda . r$ & 99.70 & r- & NAr & svq & $r \ldots v$ \\
\hline V.r & $1.0 .1 \mathrm{~V}$ & $\leqslant 1$ & var & $\Delta r \varepsilon$ & $r \ldots \Lambda$ \\
\hline ᄉ.) & $q \vee . V V$ & r.- & 191 & $\Lambda V \Lambda$ & $r \ldots q$ \\
\hline 1.7 & $9 \vee . \varepsilon r$ & ro- & $9 \vee \varepsilon$ & $9 \leqslant 9$ & $r \cdot 1$. \\
\hline 1.9 & $97 . \vee 1$ & $r \varepsilon-$ & $1 . r 0$ & $1 \ldots 1$ & $r .11$ \\
\hline 9.1 & $97 . V \pi$ & ro- & $1 . V T$ & $1 . r v$ & $r \cdot I r$ \\
\hline $1 . r$ & 90.90 & $0 .-$ & ITrV & $111 \mathrm{~V}$ & $r .1 T$ \\
\hline $1 \leq . V$ & qV.r० & ro- & ITYK & ITAV & $r \cdot 1 \varepsilon$ \\
\hline $1 . .7$ & ס. & $94-$ & 1 1ro & IrqT & $r .10$ \\
\hline 1.1 & 94.04 & $\Lambda V-$ & $1 \pi \leqslant 0$ & IrON & $r .17$ \\
\hline 1. & $q r . \cdot v$ & $9 \vee-$ & $1 \leqslant \ldots$ & $1 \pi \cdot r$ & T.IV \\
\hline $11 . \varepsilon$ & 97.91 & $\leqslant 9-$ & $17 Y \varepsilon$ & 10V0 & $r .11$ \\
\hline $1 \cdot . v$ & $9 \vee . \wedge 1$ & 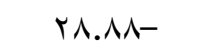 & $1117 . \varepsilon$ & 1.AV.or & المتوسط \\
\hline
\end{tabular}

المصر : وزارة الزراعة واستصلاح الأراضي، قطاع الثئون الاقتصادية، الإدارة المركزية للاقتصاد الزراعي، نثرة الميزان الغذائي، أعداد مختلفة.

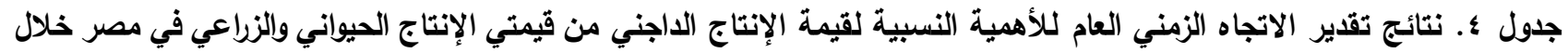

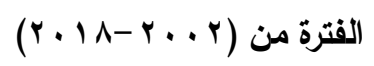

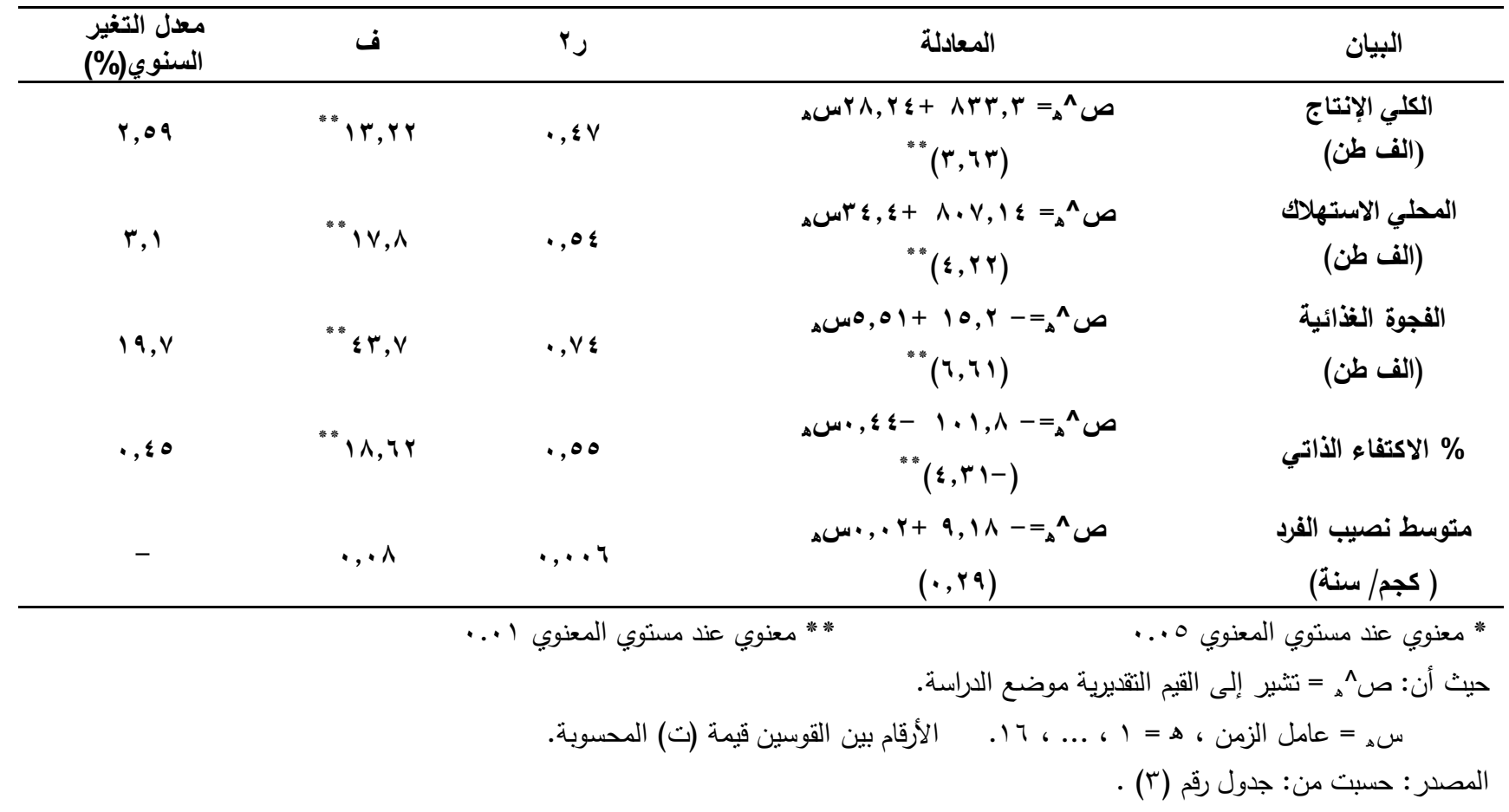


بالكيلوجرام(X) ، الاستهالاك الفردى من الاسماك بالكيلوجرام(X) )

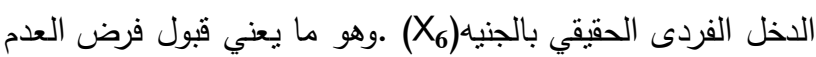

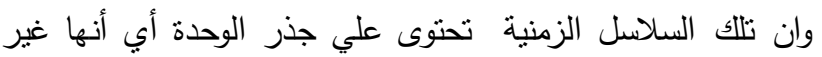

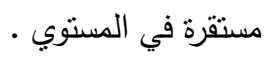
كما تبين أن القيم الاحتمالية لاختبار (PP), للنموذج الأمثل تقل عن

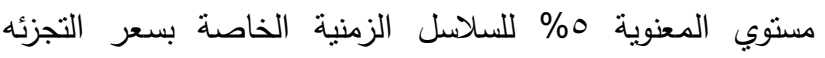

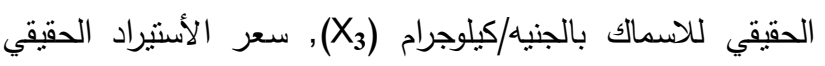
للاواجن بالجنيه/كيلوجرام (X7) , مما يعني رفض فرض العدم وان

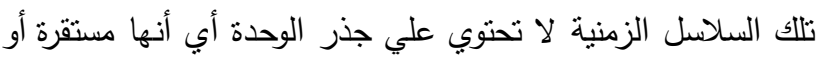
ساكنه في المستوي (Io). (10) ويتطلب تحويل السلاسل الزمنية غير الساكنة إلي سلاسل ساكنة إجراء الفروق الأولي لتنلك السلاسل الزمنية ثم إعادة اختبار

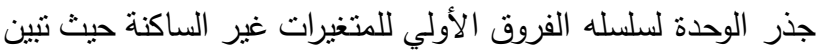

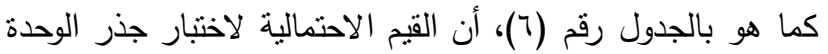

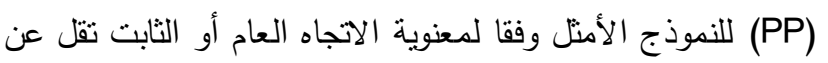

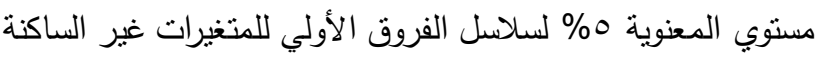

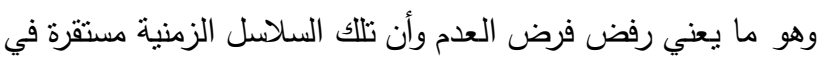

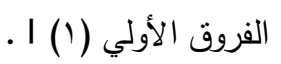

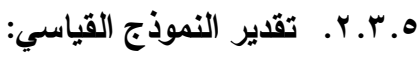

لتقلير داله الطلب علي الدواجن خلال فتره الدراسة في

الصورة اللوغاريتميه المزدوجه، تم تقلير العلاقة الانحداريه بين

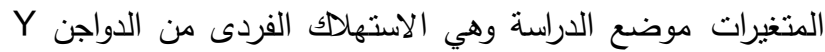

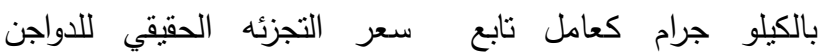
بالجنيد/كيلوجرام (X) بالجنيه/كيلوجرام بالجنيه/كيلوجرام

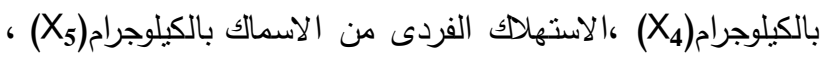

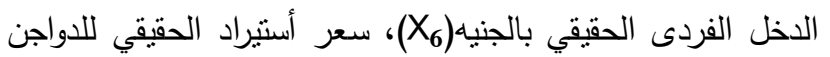
بالجنيد/كيلوجرام (XR7) كعوامل مستقلة، وذللك باستخدام نموذج ARDL والذي ينين من الثكل رقم (!) أن النموذج الأمنل لتقلير

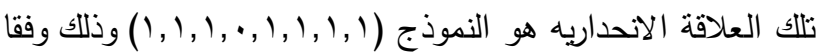
لمعيار Akaike information criteria، حيث يمثل النموذج ذو لون القيمة الأقل لهذا المعيار.
ه. ץ.0. تطور متوسط نصيب الفرد من اللحوم البيضاء: يوضح الجدول رقم (r) أن متوسط نصيب الفرد من اللحوم البيضاء

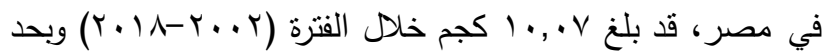

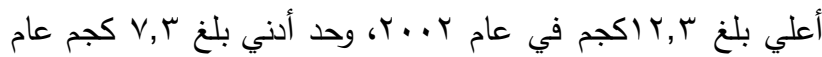

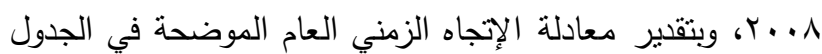
رقم (£) أن متوسط نصيب الفرد من اللحوم البيضاء أخذ اتجاهاً عاماً متزايدا لم تثبت معنويته إحصائياً. ه.r. تقدير النموذج القياسي (ARDL) لالة الطلب على اللحوم في مصر : يتضمن هذا الجزء من الدراسة تقيرا للنموذج القياسي لدالة

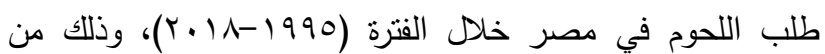

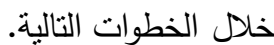

ه.ץ.1. دراسة استقرار السلاسل الزمنية للمتغيرات موضع

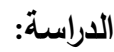

يعتمد صحة تقدير النماذج القياسية علي افتراض توافر الخصائص الإحصائية للسلاسل الزمنية المستخدمة في تقدير النموذج ، والتي يعني توافرها أن السلاسل الزمنية سلاسل مستقرة ، لإنيل

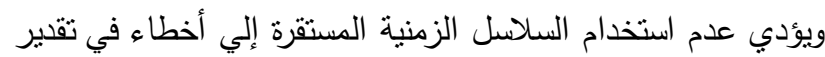

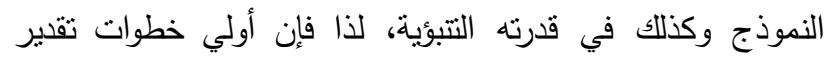
النموذج القياسي هو الكثف عن مدي استقرار وسكون السلاسل الزمنية موضع الدراسة. ستعتمد الدراسة للكثف عن استقرار وسكون السلاسل الزمنية علي اختبارات جذر الوحدة، وذلك من خلا اختبار فيلبس

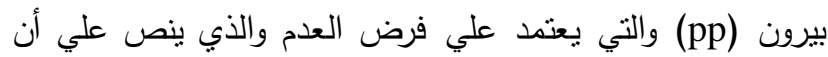
السلسلة الزمنية للمتغير غير ساكنه (يوجد فيها جذر الوحدة) مقابل الفرضية البيلة التي ينص علي أن السلسلة الزمنية للمتغير ساكنه (لا يوجد فيها جذر الوحدة)، ويتطلب إجراء اختبار استقرار السلاسل الزمنية الاعتماد علي تحليد فترات الإبطاء الزمني حيث تم الاعتماد الأبراد علي معيار Akaike information criteria لتحليد فترات الإبطاء المنلي.

وباستعراض النتائج الواردة في الجدول رقم (0) والذي يوضح نتائج اختبارات جذر الوحدة للتنغيرات موضع الدراسة، تنين أن القيم

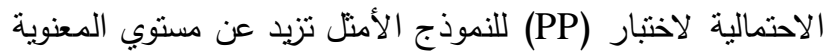
\% للسلاسل الزمنية الخاصة بسعر التجزئه الحققي للاواجن بالجنيد/كيلوجرام (X) بالجنيه/كيلوجرام 
جدول ه. نتائج اختبار استقرار السلاسل الزمنية في المستوي لمتغيرات داله الطلب علي الدواجن باستخدام اختبار جذر الوحدة خلال الفترة

\begin{tabular}{|c|c|c|c|c|}
\hline \multirow{3}{*}{ القرار } & \multicolumn{4}{|c|}{.$(r+11-1990)$} \\
\hline & \multicolumn{3}{|c|}{ PP } & \multirow{2}{*}{ الاختبار } \\
\hline & بدون ثابت واتجاه عام & ثابت فقط & ثابت واتجاه عام & \\
\hline ق ق قبول فرض العدم & $\begin{array}{l}* 1 . .9 \\
(. .91)\end{array}$ & $\begin{array}{c}1.7- \\
(\cdot .80)\end{array}$ & $\begin{array}{l}1.91- \\
(. .0 \mathrm{~V})\end{array}$ & $\mathbf{Y}$ \\
\hline ق ق قبول فرض العدم & $\begin{array}{l}* 1,11 \\
(\cdot .7)\end{array}$ & $\begin{array}{c}1, Y- \\
(\cdot .70)\end{array}$ & $\begin{array}{c}1, Y r \\
(\cdot . \wedge \wedge)\end{array}$ & $\mathbf{x} 1$ \\
\hline قبول فرض العدم & $\begin{array}{l}* \varepsilon . .0 \\
(. .99)\end{array}$ & $\begin{array}{c}r .0 \\
(. .99)\end{array}$ & $\begin{array}{c}. . r r \\
(. .99)\end{array}$ & $\mathbf{X} 2$ \\
\hline رفض فرض العدم والسلسله (0) I & - & $\begin{array}{l}* \% \varepsilon . V- \\
(\cdots \cdot 1)\end{array}$ & $\begin{array}{c}0 . \varepsilon- \\
(\cdots, r)\end{array}$ & $\mathbf{X 3}$ \\
\hline ق ق قبول فرض العدم & - & -- & $\begin{array}{l}\text { *Y.YV- } \\
(\cdot Y Y)\end{array}$ & $\mathrm{X} 4$ \\
\hline ق ق قبول فرض العدم & -_ & $\begin{array}{c}* . r 1- \\
(\cdot . r)\end{array}$ & $\begin{array}{l}. . r Y- \\
(.94 \wedge)\end{array}$ & $\times 5$ \\
\hline قبول فرض العدم & $\begin{array}{l}* 1 . .4 \\
(. .91)\end{array}$ & $\begin{array}{c}. . v_{0} \\
(. .11)\end{array}$ & $\begin{array}{l}r .1 \leq- \\
(\cdot .0)\end{array}$ & X6 \\
\hline رفض فرض العدم والسلسله (0) I & - & $\begin{array}{l}*^{*} \mathrm{r} .19- \\
(\ldots . \mathrm{r})\end{array}$ & $\begin{array}{c}\text { «.०- } \\
(\cdots \cdot v)\end{array}$ & $\times 7$ \\
\hline
\end{tabular}

المصدر : جمعت وحسبت من : جدول ( ' () بالملحق.

جدول ؟. نتائج اختبار استقرار السلاسل الزمنية في الفروق الأولي لمتغيرات داله الطلب علي الدواجن باستخدام اختبار جذر الوحدة خلال

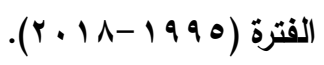

\begin{tabular}{|c|c|c|c|c|}
\hline \multirow{2}{*}{ القرار } & \multicolumn{3}{|c|}{$P p$} & \multirow{2}{*}{ الاختبار } \\
\hline & بدون ثبات واتجاه عام & ثبات فقط & ثبات واتجاه عام & \\
\hline رفض فرض العدم والسلسله (1) I & $\begin{array}{l}* \varepsilon .1- \\
(\cdots \ldots)\end{array}$ & $\begin{array}{c}\varepsilon \cdot r- \\
(\cdots r)\end{array}$ & $\begin{array}{l}\varepsilon . \mid V- \\
(\ldots . \mid V)\end{array}$ & $Y$ \\
\hline رفض فرض العدم والسلسله (1) I & $\begin{array}{l}* r, \Upsilon- \\
(\cdot,, r)\end{array}$ & $\begin{array}{l}r, r- \\
(\cdot, \cdot r)\end{array}$ & $\begin{array}{l}r, Y- \\
(\cdot, \cdot 9)\end{array}$ & $X_{1}$ \\
\hline رفض فرض العدم والسلسله (1) I & - & - & $\begin{array}{l}*\lfloor. \mid V- \\
(\ldots, \mid V)\end{array}$ & $X_{2}$ \\
\hline رفض فرض العدم والسلسله (1) & $\begin{array}{l}* 0.7- \\
(\cdot, \ldots)\end{array}$ & $\begin{array}{c}0.7- \\
(\cdot, \cdots r)\end{array}$ & $\begin{array}{c}0 . \leqslant V- \\
(\cdot, \ldots 1)\end{array}$ & $\mathbf{X}_{4}$ \\
\hline رفض فرض العدم والسلسله (1) & - & $\ldots$ & $\begin{array}{l}* \ldots, 0- \\
(\cdot, \cdot r)\end{array}$ & $X_{5}$ \\
\hline رفض فرض العدم والسلسله (1) & $\begin{array}{l}* 0.1 \mathrm{r-} \\
(\cdots \ldots)\end{array}$ & $\begin{array}{c}0 .\{- \\
(\cdots \cdots)\end{array}$ & $\begin{array}{c}0 . r- \\
(\cdots, 1)\end{array}$ & $x_{6}$ \\
\hline
\end{tabular}


Akaike Information Criteria (top 20 models)

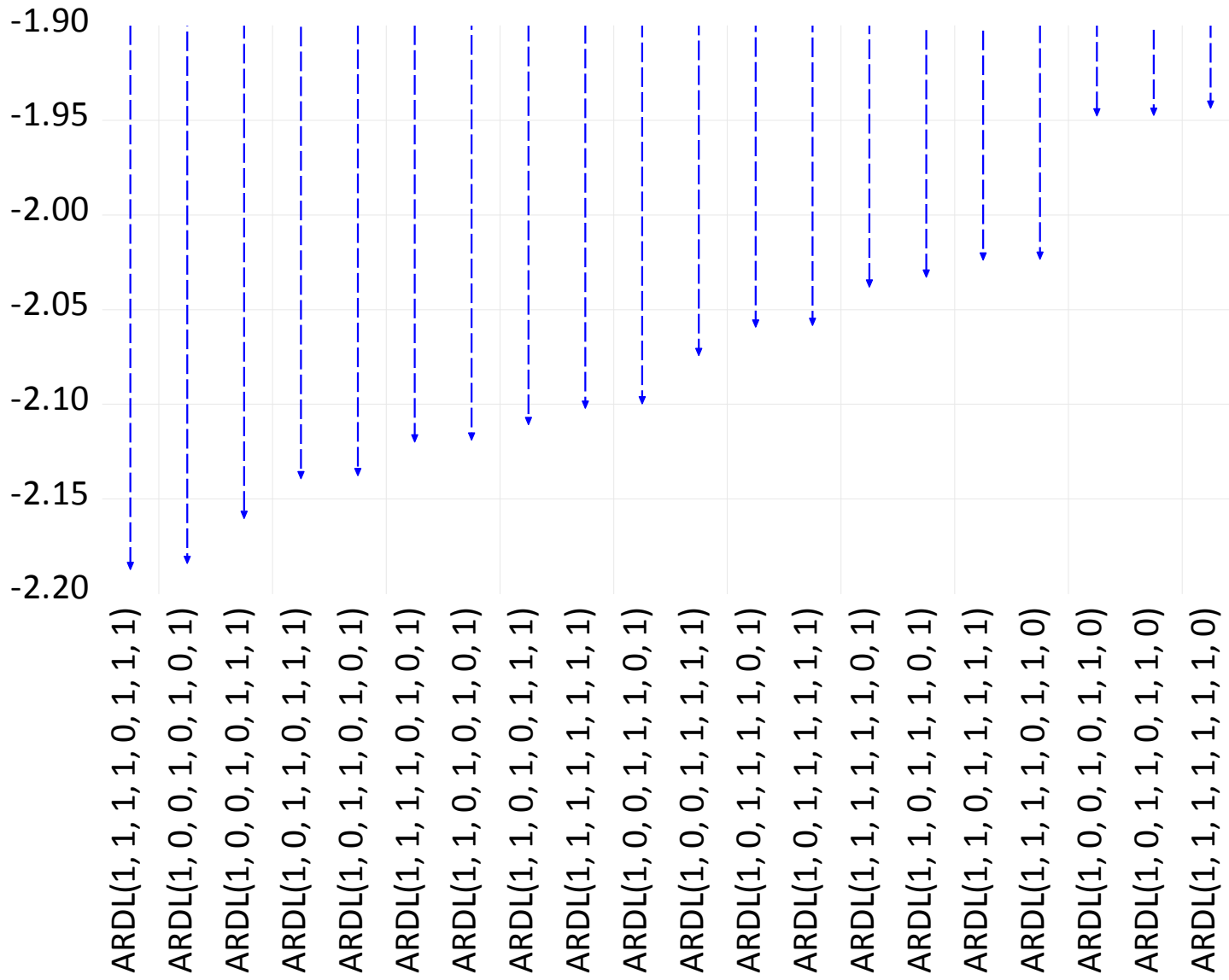

Akaike information Criteria الثكل 1. أختيار نموذج ARDL المثل وفقاً لمعيار

ومن خلال تقلير هذا النموذج يمكن الكثف عن التكامل المشترك المتغيرات وفقا لنموذج (Ardl) ، حيث تثنير نتائج الاختبار

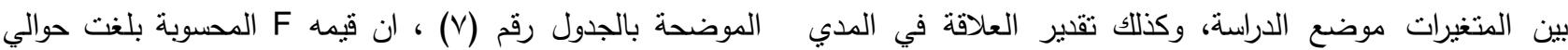

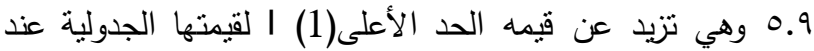
الطويل والمدي القصير كما يلي: مستوي معنويه (\%)، وهو ما يؤكد وجود علاقة نوازنية طويلة الأجل أ - الكثف عن التكامل المشترك: بين المتغيرات في النموذج. bounds test من خلال اختبار الحدود للتكامل المشترك يتم تقلير إحصائية f-bounds test لاختبار التكامل المشترك بين

جدول V. نتائج اختبار الحدود للتكامل المشترك

\begin{tabular}{|c|c|c|}
\hline \multicolumn{2}{|c|}{0.9} & قيمه F \\
\hline \multicolumn{2}{|c|}{ القيم الجدولية } & \multirow{2}{*}{ ستتوي المعنوية } \\
\hline العدود الدنيا (0) I & الحدود العليا (1) I & \\
\hline$r, r V$ & $r, \leqslant q$ & $\% 1$. \\
\hline T.VT & א.17 & $\% 0$ \\
\hline$\varepsilon, Y \Lambda$ & $0, \wedge \varepsilon$ & $\% 1$ \\
\hline
\end{tabular}


الدراسة، بالإضافة إلي أنه يوضح سرعه التعليل نحو النوازن في

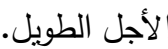

وقد أوضحت النتائج الموضحة في الجدول رقم (^)، أن

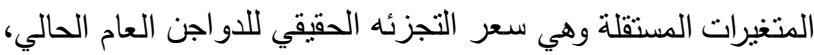
سعر التجزئه الحقيقي للحوم الحمراء العام الحالي، و سعر التجزئه الحقيقي للاسماك العام الحالي، كميه الاستهلاك الفردي العام الحالي

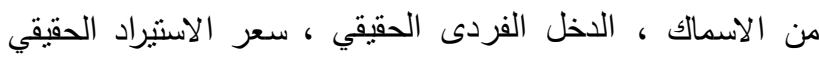

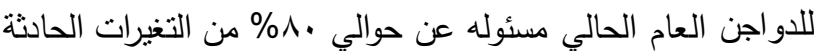
في كميه استهالك الفردي من اللحوم الحمراء في الأجل القصبر.

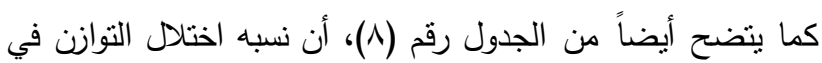

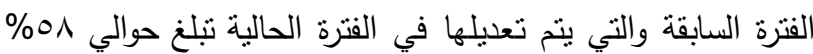
خلال العام الواحد في اتجاه مستوي التوازن المرغوب له في المدي

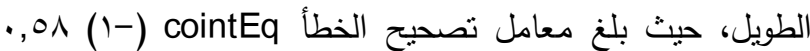

ب - تقدير العلاقة بين المتغيرات في المدي الطويل: بعد التأكد من وجود التكامل المثترك بين متغيرات داله طلب اللحوم الحمراء ، أي التأكد من وجود علاقة توازنيه في المدي الطويل بين

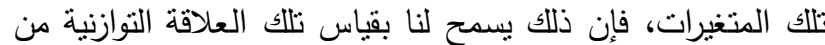

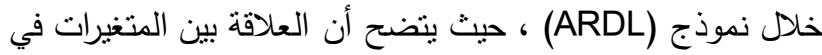
المدي الطويل يمكن تمثيلها بالمعادلة التالية: $\ln Y=6.17-0.85 \ln X_{1}-0.04 \ln X_{2}+.0 .26 \ln X_{3}$ $0.57 \ln \mathrm{X}_{4}+0.43 \ln \mathrm{X}_{5}-0.17 \ln \mathrm{X}_{6}-0.36 \ln \mathrm{X}_{7}$ $(-2.9) *$
$(-0.25)$
$(2.46) *$

حيث يتضح معنويه تأثير سعر التجزئه الحقيقي للاواجن العام الحالي (X), سعر التجزئه الحقيقي للاسماك العام الحالي(X) علي كميه الاستهلاك الفردي من الدواجن وذللك عند مستوي معنوية 0\% ج-تقدير العلاقة في المدي القصير:

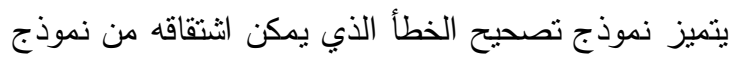
بأنه يمنا بالعلاقة قصبرة الأجل بين المتغيرات موضع (ARDL)

جدول ^. نتائج تقدير العلاقة في المدي القصير من خلال نموذج تصحيح الخطأ.

\begin{tabular}{|c|c|c|c|}
\hline sig الاحتمال & $\mathrm{t}$ & المعلمات & المتغير \\
\hline.$Y T$ & $1 . Y-$ & $. .1 \mathrm{~V}-$ & $\ln X_{1}$ \\
\hline$\ldots . .0$ & r.10 &.$r \varepsilon$ & $\ln X_{2}$ \\
\hline$\cdots \cdots$ & $0.7 \mathrm{~V}$ &. .0 & $\ln X_{3}$ \\
\hline$\cdots r$ & 纟.५- &..$\leq q-$ & $\ln X_{5}$ \\
\hline$\cdots \varepsilon \varepsilon$ &..$\wedge 1$ & $\cdots 7$ & $\ln X_{6}$ \\
\hline$\ldots r$ & r.O- & $\cdots \wedge-$ & $\ln X_{7}$ \\
\hline \multirow[t]{3}{*}{ صفر } & 9.V- & $. .01-$ & cointEq(-1) \\
\hline & \multicolumn{2}{|c|}{ المؤشرات الإحصائية } & \\
\hline & & & $\mathbf{R 2}$ \\
\hline
\end{tabular}

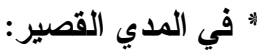

•بلغت مرونة الطلب الثقاطعية بالنسبة لسعر التجزئه الحقيقي للحوم الحمراء العام الحالي حوالى \& ـ.. ، أى ان زياده سعر التجزئه الحققى للحوم الحمراء بمقدار ·(\% يؤدى إلى زياده كمية

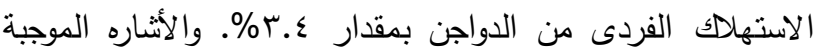
للمرونه التقاطعية تدل على ان السلعتين اللحوم الحمراء والدواجن

$$
\text { بدائل لبعضهما فى نفس العام. }
$$

هلغت مرونة الطلب التقاطعية بالنسبة لسعر التجزئه الحقيقى للاسماك العام الحالي حوالى ه... ، بأى ان زياده سعر التجزئه الحقيقى للاسماك بمقدار • \% يؤدى إلى زياده كمية الاستهالاك الفردى من الدواجن بمقدار 0.•\%. والأشاره الموجبة للمرونه

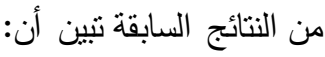
* في المدي الطويل : • بلغت مرونة الطلب السعرية للدواجن حوالى -10. · وهى سالبة

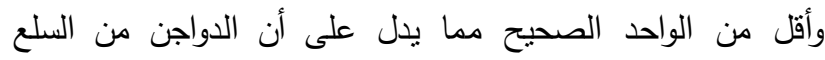

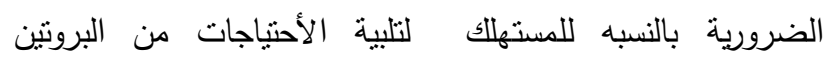

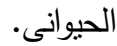
•بلغت مرونة الطلب التقاطعية بالنسبة لسعر التجزئه الحقيقى للاسماك حوالى بr.. ، ،أى ان زياده سعر التجزئه الحقيقى للاسماك بمقدار •1\% يؤدى إلى زياده كمية الاستهلاك الفردى من الدواجن

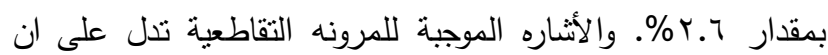
السلعتين الدواجن والاسماك بدائل لبعضهما فى نفس العام. 


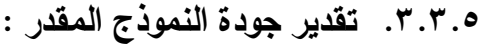

لابد من اختبار الاستقرار الهيكلي لمعاملات الأجل القصير

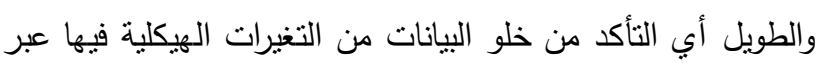

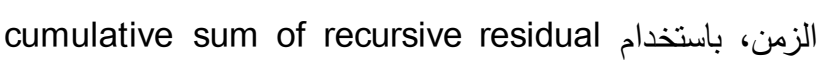
حيث يتضح كما هو موضح بالثكل رقم (Y) أن (CUSUM) الثكل اليياني الإحصائي (CUSUM) يقع داخل الحدود الحرجة عند مستوي معنويه 0\%. الأمر الذي يعني تحقق الاستقرار الهيكلي

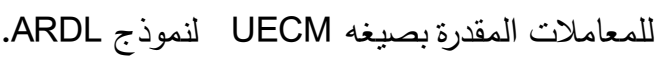

التقاطعية تدل على ان السلعتين الدواجن والاسماك بدائل لبعضهما

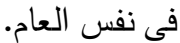

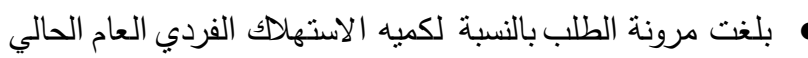

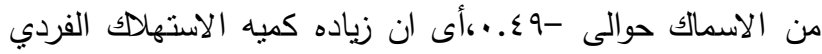

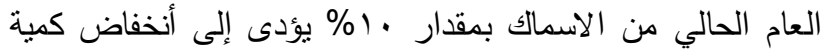
الاستهلالك الفردى من الدواجن بمقدار 9.؛ \%، وذلك يؤكد النتيجه السابقة حيث تعتبر الدواجن والاسماك بدائل لبعضهم.

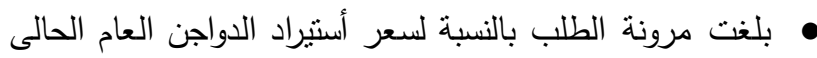
حوالى -1. ... ، مما يدل على أنه طلب غير مرن حيث أن زيادة سعر الأستبراد بمقدار (\% يؤدى إلى خفض الكمية المطلوبة بمقدار $. \% \cdot . \wedge$

1.6

1.2

0.8

0.4

O.O

$-0.4$

17

18

19

20

21

22

23

24

CUSUM of Squares

$5 \%$ Significance

شكل r. اختبار (cusum) للكثف عن الاستقرار الهيكلي للمعاملات المقدرة بصيغه UECM لنموذج ARDL.

Breusch-Pagan-) وفقا لاختبار F الاحتمالية لإحصائية Godfrey

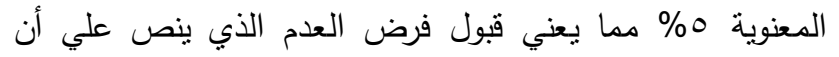
البواقي متجانسة. • ما نتير البيانات الموضحة في نفس الجدول والموضحة في الثكل رقم (ए)، أن البواقي موزعها نوزيعا طييعيا حيث بلغت القيمة

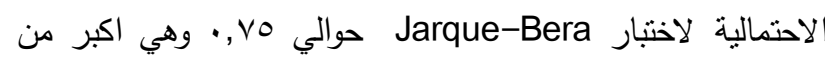

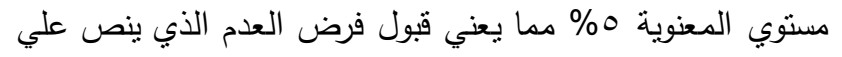
عدم احتواء البواقي المقدرة من النموذج علي مشكله التوزيع الطبيعي.
كما تم إجراء الاختبارات القياسية كما هو موضح في الجدول رقم (9) للحكم علي جوده النموذج وخلوه من المشاكل القياسية المختلفة، حيث نتير نتائج نلاك الاختبارات ان النموذج المقدر خالي من مشكله

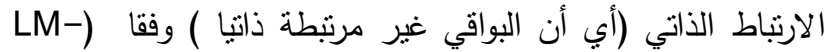
حيث بلغت القيمة الاحتمالية ل F حوالي reST

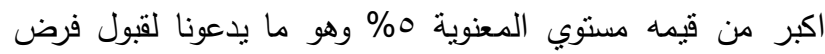

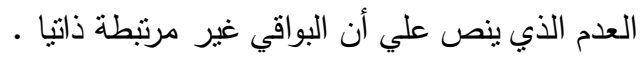
• كما تثير الييانات الموضحة لنفس الجدول إلي أن البواقي

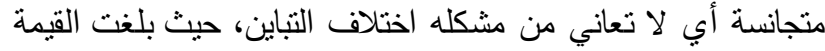
ARDL جدول 9.

\begin{tabular}{|c|c|c|c|}
\hline القيمة الاحتمالية & القيمة & إحصائية & الاختبار \\
\hline$\cdot .1 \cdot r$ & r.s & $\mathbf{F}$ & الارتباط الذاتي (LM-TEST) \\
\hline$\cdot$, ov & $\therefore .94$ & $\mathbf{F}$ & اختلاف التباين (Breusch-Pagan-Godfrey) \\
\hline.$\vee \vee \varepsilon$ &. $.0 \mathrm{~V}$ & Jarque - Bera & التوزيع الطبيعي (Jarque-Bera) \\
\hline
\end{tabular}



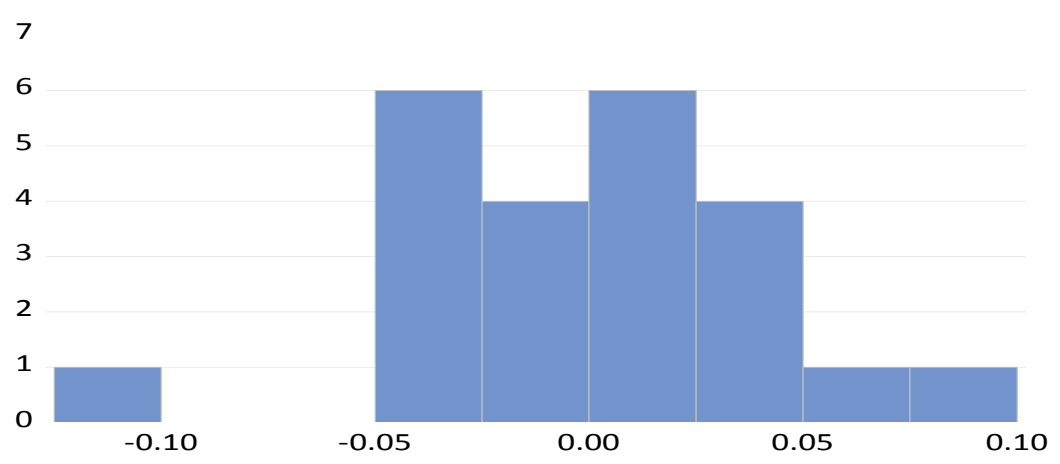

\begin{tabular}{|lr|}
\hline \multicolumn{2}{|l|}{ Series: Residuals } \\
Sample 2 24 & \\
Observations 23 \\
Mean & $6.18 \mathrm{e}-16$ \\
Median & 0.000914 \\
Maximum & 0.082886 \\
Minimum & -0.107288 \\
Std. Dev. & 0.043220 \\
Skewness & -0.385926 \\
Kurtosis & 3.086619 \\
& \\
Jarque-Bera & 0.578121 \\
Probability & 0.748967
\end{tabular}

$$
\text { شكل r. اختبار التوزيع الطبيعي النموذج. }
$$

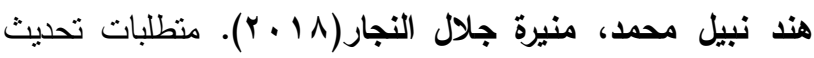

التسويق المحلي للدواجن في مصر، المجلة المصرية للاقتصاد

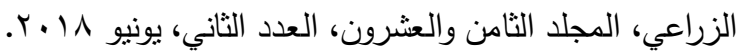

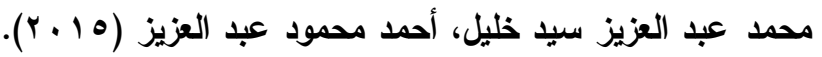

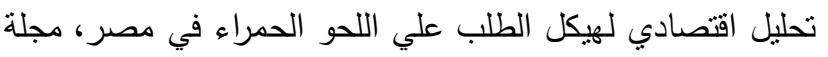

المنصور للعلوم الزراعية، كلية الزراعة، جامعة المنصورة، المجلد جا،

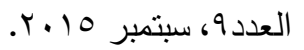

\section{REFERENCES}

Enders Walter (2001). Applied Econometric Time Series, fourth ed. New York: Wiley, 2001.

Gujarati, Damodar N, Basic Econometrics (2004). Fourth Edition, Mc Graw- Hill, 1 nc, 2004.

Pesaran MH, Shin Y, Smith RJ (2001). Bound testing approaches to the analysis of level relationships. Journal of Applied Econometrics, 2001.

Phillips PCB, Perron P (1988). Testing for a unit root in time series regression. Biometrika, Vol. 75, No. 2, pp (335-346).

وعلى ذللك يوصى البحث بضرورة الأهتمام بمشاريع الدواجن لتوفيرها للمستهلكين بسعر مناسب ،وعدم اللجوء للاستيراد حيث يؤثز سعر الاستيراد على الكمية المستهلكه منها، بالاضافه إلى أنها من السلع الضرورية والتى يلجأ أليها المستهلكبن فى حالة ارتفاع أسعار اللحوم الحمراء والأسماك.

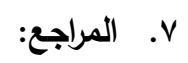

الجهاز المركزي للتعبئة العامة والأحصاء، الكتاب الاحصائي السنوي، أعداد متفرقة. الجهاز المركزي للتعبئة العامة والأحصاء، النشرة السنوية لأسعار الموارد النهائية والخدمات، أعداد مختلفة. وزارة الزراعة واستصلاح الاراضي ، قطاع الثئون الاقتصادية،

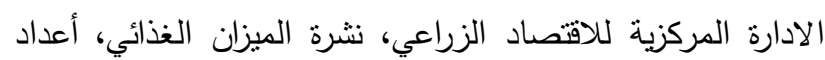

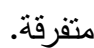

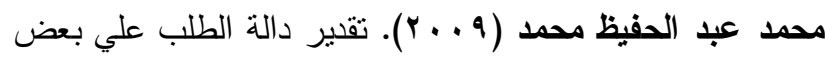
المصادر الأساسية للبروتين الحيواني في مصر، مجلة أسيوط للعلوم

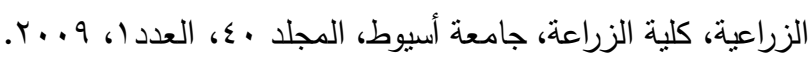




\title{
Factors Determining The Demand For white Meat in Egypt
}

\author{
Dalia Hamed El Showeikh ${ }^{1}$ and Hassan Mousa Radwan ${ }^{2}$
}

${ }^{1}$ Department of Agricultural Economics, Faculty of Agriculture, Assiut University, Assiut

${ }^{2}$ Higher Institute for Cooperation and agricultural extension in Assiut

\begin{abstract}
The research reached several results as follows:

1- There is a statis tically significant increase in both the total production available forconsumption and the nutritional gap of white meat estimated at about 28.24,34.4 and 5.51 thou sand tons, with an annual increase rate of about 2.59\% and $3.1 \%$ and respectively, as it was found that there is a statistically significant decrease in the rate of self-sufficiency of white meat estimated at about $44 \%$, with an annual decrease rate of about $0.45 \%$.

2- The optimal ARDL model for estimating this regression relationship is $(1,1,1,0,1,1,1,1)$, according to the Akaike information criteria, where it represents the model with the lowest value for this criterion.

In the long run:

- The price elasticity of demand for poultry is about -0.85 , which is negative and less than one, which indicates that poultry is one of the necessary commodities for the consumer to meet the needs of animal protein.

In the short term:

The cross elasticity of demand in relation to the real retail price of red meat this year is about 0.34 , meaning that an increase in the real retail price of red meat by $10 \%$ leads to an increase in the amount of individual consumption of poultry by $3.4 \%$. The positive sign of the cross elasticity indicates that the two commodities, red meat and poultry, are substitutes for each other in the same year.
\end{abstract}

KEYWORDS: Factors Determining, white Meat, Egypt 
المـلاحق:

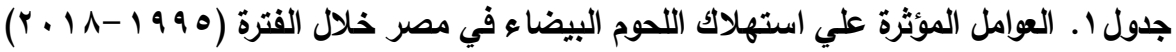

\begin{tabular}{|c|c|c|c|c|c|c|c|c|}
\hline سعر الاستيراد & 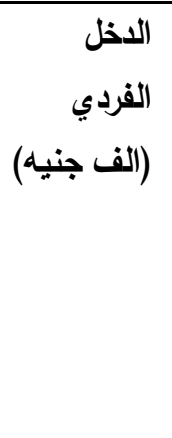 & منوسط & متوسط & 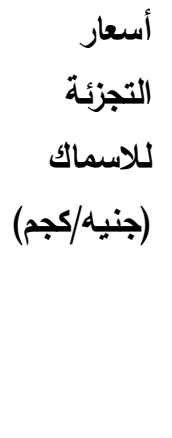 & 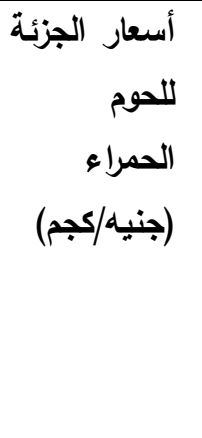 & 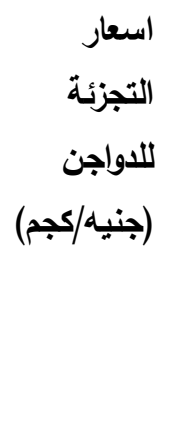 & متوسط نصيب & السنوات \\
\hline $7, \cdot 9$ & r,or & А... & 17,7 & 7,70 & $1 \leq, O r$ & $\varepsilon, 90$ & $\varepsilon \wedge$. & 1990 \\
\hline 1. & r, & $\wedge, 0$ & 17,0 & $v, r_{O}$ & $10, r_{0}$ & 0,1 . & $\varepsilon q \pi$ & 1997 \\
\hline 7,99 & $\varepsilon, \cdot \wedge$ & 9,1 & $17, r$ & $V, 70$ & $10, \leqslant T$ & $0,1 Y$ & 0.7 & 1998 \\
\hline$r, q$. & ه, & $1 \cdot, 9$ & 17,9 & $V, V Y$ & $10, \mathrm{~V} 9$ & $0, r \varepsilon$ & 011 & 1991 \\
\hline $0, Y_{0}$ & $\varepsilon, 01$ & r, & $1 \wedge, 9$ & V,Y & $17,7 V$ & 0,1 & Trq & 1999 \\
\hline 0,07 & $\varepsilon, q \pi$ & $M, \varepsilon$ & $19, V$ & $V$, ro & $0,1 Y$ & IV, r & 779 & $r \ldots$ \\
\hline $0, V V$ & $0, .9$ & $1 \leq, \varepsilon$ & $r \cdot, r$ & V,rی & $0, r$. & IV,Ir & אדג & $r \ldots l$ \\
\hline $7,7$. & $0, V_{1}$ & $1 \varepsilon, \Gamma$. & $r \varepsilon, \wedge$. & $7, \vee 9$ & $1 \wedge, \cdot r$ & 0,10 & IT.r & $r \ldots r$ \\
\hline 7,71 & $0, \vee 9$ & $10, Y \leqslant$ & $11,1$. & $\wedge, \pitchfork \wedge$ & $r \cdot, \wedge \varepsilon$ & $7, \varepsilon \varepsilon$ & $11 . r$ & $r$ \\
\hline $0, Y_{1}$ & 7,01 & 10,7 & $1 \cdot, \varepsilon$ & $1 \cdot, \cdot r$ & $r \leq, 01$ & $\wedge, \vee$. & 11.1 & $r \ldots \varepsilon$ \\
\hline$\wedge, \cdot \varepsilon$ & $V, 17$ & 10,r & 11,1 . & $\Lambda, 7)$ & $r T, \wedge T$ & V,r. & $1 \cdot v$ & r. . o \\
\hline$V, V Y$ & $1, .0$ & IT, & Ir, V. & 9,0 & $r q, r v$ & $\Lambda, T V$ & $\vee . \wedge$ & $r . . r$ \\
\hline$\wedge, Y T$ & $q, T_{1}$ & 17,91 & $1 r, \ldots$ & $1 \cdot, \wedge T$ & דז,Yז & $\Lambda, T r$ & A.r & $r \ldots r$ \\
\hline $1 \cdot, \wedge \wedge$ & $9, \leq 0$ & 10,90 & $1 \cdot, 9$ & $1 \cdot, \lambda 1$ & דז,דr & $11,9 \leq$ & v.r & $r . \lambda$ \\
\hline$\wedge, \wedge \wedge$ & $1 \cdot, 15$ & 10,19 & $1 \cdot, 9$. & $M, \cdot T$ & $\varepsilon \cdot, \Sigma \wedge$ & $1 r_{,} \cdot 1$ & ^.) & $r . . q$ \\
\hline $9,9 \vee$ & $1 \cdot, 91$ & $19, \mathrm{~V}$ & $q, \wedge$. & IT, & מיט, & $1 \leqslant, \pi r$ & A. 7 & $r \cdot 1$. \\
\hline דו, 10, & סr, 11 & $19, .9$ & $q, \varepsilon$. & $1 \varepsilon, \cdot r$ & O^, T. & זד, 17 & 1.9 & $r .11$ \\
\hline 19,19 & $1 \%, \varepsilon 7$ & $r \cdot, \infty 0$ & $q, r$. & iv, & $7 r, q$ & $r \cdot, r$ & 9.1 & $r .1 T$ \\
\hline ^, & $\mid \varepsilon, r \varepsilon$ & 19,Vr & $q, v$. & $r \cdot, r r$ & $7 V, 10$ & rד, rr & $1 \cdot r$ & $r .1 r$ \\
\hline IT,V & $r \wedge, \wedge r$ & $r \cdot, \wedge r$ & $1 \cdot, 1$. & rr, VA & $V V, q \leq$ & $r \leqslant, q r$ & $1 \varepsilon . V$ & $r .1 \leq$ \\
\hline 14,70 & r.,o. & $r \cdot, 1 \wedge$ & $11, \varepsilon$. & & 17,79 & $r \leq, 0$. & $1 \cdot .7$ & $r .10$ \\
\hline $17, r$. & $\Gamma \varepsilon, \wedge$. & $r 1,7 \varepsilon$ & $q, r$. & $r, \wedge)$ & $9 \vee, 7 \vee$ & rV, qr & 1.1 & $r .17$ \\
\hline$r \cdot, \wedge$ & $\varepsilon \varepsilon, Y Y$ & r Y, VT & $1 \cdot, v \cdot$ & ו, & سז,q & rr,qr & 1. & $r .1 \mathrm{~V}$ \\
\hline rT, rV & or,, . & 17,0 & $1 \cdot, r$ & YA, VV & $1 \leq r, q r$ & $r v, v T$ & $11 . \varepsilon$ & $r \cdot 11$ \\
\hline
\end{tabular}

1- الجهاز المركزي للتعبئة العامة والاحصاء، الكتاب الاحصائي السنوي، إعداد متقرقة. r - وز ارة الزر اعة و استصلاح الأر اضي، قطاع الشئئون الاقتصادية، الإدارة المركزية للاقتصاد الزر اعي، نشرة الميزان الغذائي، أعداد متفرقة. 